\title{
The Reduction in Gastric Atrophy after Helicobacter pylori Eradication Is Reduced by Treatment with Inhibitors of Gastric Acid Secretion
}

\author{
Ryota Niikura ${ }^{1, * \mathbb{D}}$, Yoku Hayakawa ${ }^{1, * \mathbb{D}}$, Yoshihiro Hirata ${ }^{1}$, Keiji Ogura ${ }^{2}$, \\ Mitsuhiro Fujishiro ${ }^{1,3}$, Atsuo Yamada ${ }^{1}$ (D), Tetsuo Ushiku ${ }^{4}$, Mitsuru Konishi ${ }^{1}$, \\ Masashi Fukayama ${ }^{4}$ and Kazuhiko Koike ${ }^{1}$ \\ 1 Department of Gastroenterology, Graduate School of Medicine, The University of Tokyo, 7-3-1 Hongo, \\ Bunkyo-ku, Tokyo 113-8655, Japan; HIRATAY-INT@h.u-tokyo.ac.jp (Y.H.); mtfujish-kkr@umin.ac.jp (M.F.); \\ yamada-a@umin.ac.jp (A.Y.); konishi524326@gmail.com (M.K.); kkoike-tky@umin.ac.jp (K.K.) \\ 2 Department of Gastroenterology, Tokyo Metropolitan Police Hospital, Tokyo 164-8541, Japan; \\ keiji-tky@umin.ac.jp \\ 3 Department of Gastroenterology \& Hepatology, Nagoya University Graduate School of Medicine, \\ Nagoya 466-8560, Japan \\ 4 Department of Pathology, Graduate School of Medicine, The University of Tokyo, Tokyo 113-8655, Japan; \\ usikut@gmail.com (T.U.); mfukayama-tky@umin.ac.jp (M.F.) \\ * Correspondence: niikura-dky@umin.ac.jp (R.N.); yhayakawa-tky@umin.ac.jp (Y.H.); \\ Tel./Fax: +81-3-3815-5411 (R.N. \& Y.H.)
}

Received: 5 March 2019; Accepted: 16 April 2019; Published: 18 April 2019

\begin{abstract}
Background: Helicobacter pylori (H. pylori) eradication therapy may improve gastric atrophy and intestinal metaplasia, but the results of previous studies have not always been consistent. The aim of this study was to compare the histological changes of intestinal metaplasia and gastric atrophy among the use of acid-suppressing drugs after H. pylori eradication. Methods: A cohort of 242 patients who underwent successful eradication therapy for $H$. pylori gastritis and surveillance endoscopy examination from 1996 to 2015 was analyzed. Changes in the histological scores of intestinal metaplasia and atrophy according to drug use (proton-pump inhibitors (PPIs), $\mathrm{H}_{2}$ receptor antagonists (H2RAs), and non-acid suppressant use) were evaluated in biopsies of the antrum and corpus using a generalized linear mixed model in all patients. Results: The mean follow-up period and number of biopsies were $5.48 \pm 4.69$ years and $2.62 \pm 1.67$ times, respectively. Improvement in the atrophy scores of both the antrum $(p=0.042)$ and corpus $(p=0.020)$ were significantly superior in patients with non-acid suppressant drug use compared with those of PPI and H2RA use. Metaplasia scores in both the antrum and corpus did not improve in all groups, and no significant differences were observed among groups in the antrum $(p=0.271)$ and corpus $(p=0.077)$. Conclusions: Prolonged acid suppression by PPIs or H2RAs may limit the recovery of gastric atrophy following H. pylori eradication.
\end{abstract}

Keywords: H. pylori eradication; atrophy; proton pump inhibitors (PPIs)

\section{Introduction}

H. pylori eradication therapy may have the potential to regress atrophic and metaplastic changes and subsequently prevent the development of gastric cancer. In fact, previous studies have suggested that H. pylori eradication reduces metachronous recurrence of gastric cancer after endoscopic surgery [1-5]. It has been suggested that $H$. pylori eradication partly improves atrophy and metaplasia as well [6], although there are numerous reports which failed to demonstrate such histological reversion, and indeed the improvement of metaplasia after eradication has not been confirmed by meta-analyses. 
Previous studies have reported that oxyntic atrophy seems to be a pathogenic factor in gastric carcinogenesis [7-9]. Reduced gastric acidity in the atrophic stomach leads to hypergastrinemia, which stimulates the function and proliferation of enterochromaffin-like cells located in the oxyntic mucosa, and potentially promotes gastric carcinogenesis in the oxyntic mucosa [10]. Recently, we and others have reported that long-term use of proton pump inhibitors (PPIs) may be a risk factor for gastric cancer after H. pylori eradication [11,12]. It is well known that acid suppression by PPIs and other drugs causes hypergastrinemia via a negative feedback mechanism, which may be associated with the subsequent risk of gastric cancer [10]. In contrast, the role of metaplasia in gastric carcinogenesis has been questioned in recent years, and metaplasia may just be a marker of long-term atrophic gastritis $[8,9,13,14]$.

In this report, we conducted a retrospective cohort study which collected clinical and histological data from $\mathrm{H}$. pylori-eradicated patients. We quantitatively evaluated the reversibility of histological findings of intestinal metaplasia, atrophy, and inflammation using the updated Sydney system, and investigated the associations with several clinical and molecular parameters, focusing especially on the use of acid-suppressing drugs.

\section{Results}

\subsection{Patients}

A total of 242 eligible patients who underwent successful eradication therapy for H. pylori gastritis were analyzed (Figure 1A, study flow chart). The baseline characteristics of the patients by drug use are shown in Table 1. PPI, $\mathrm{H}_{2}$ receptor antagonists (H2RA), and non-acid suppressant drug use were $110(45.45 \%), 33(13.64 \%)$, and 99 (40.91\%) patients, respectively. The mean durations of PPI and H2RA use were 915 days and 838 days, respectively. No significant differences in baseline characteristics were observed among drug users, except for non-steroidal anti-inflammatory drug (NSAID) use $(p<0.001)$. The mean follow-up period was $5.48 \pm 4.69$ years.

(A)

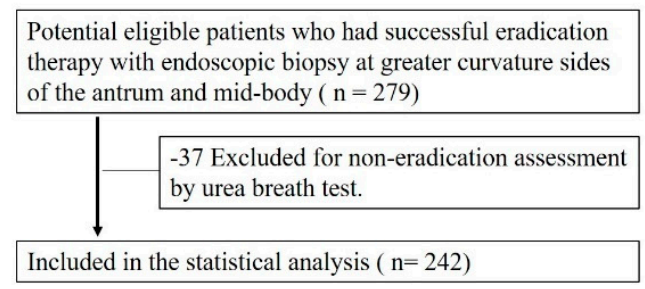

(B)

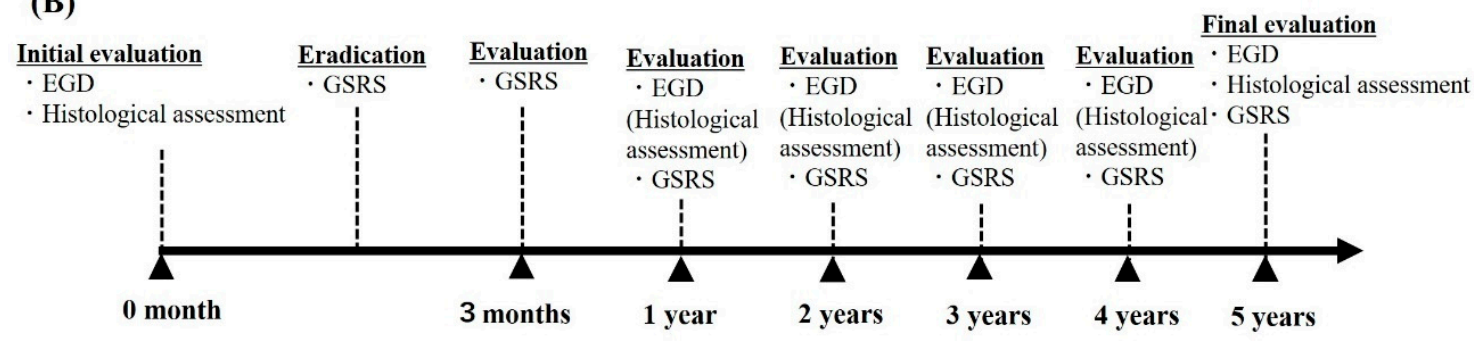

Figure 1. (A) Study flow chart; (B) Study time course. Abbreviation: EGD, Esophagogastroduodenoscopy; GSRS, gastrointestinal symptom rating scale. 
Table 1. Baseline patient characteristics by drug use $(n=242)$.

\begin{tabular}{|c|c|c|c|c|}
\hline \multirow[b]{2}{*}{ Characteristics } & \multicolumn{3}{|c|}{ No. of Patients (\%) } & \multirow[b]{2}{*}{$p$ Value } \\
\hline & $\begin{array}{l}\text { PPI Use } \\
(n=110)\end{array}$ & H2RA Use $(n=33)$ & $\begin{array}{c}\text { Non-Acid Suppressant } \\
\text { Use }(n=99)\end{array}$ & \\
\hline \multicolumn{5}{|l|}{ Age category (years) } \\
\hline$<50$ & $4(3.63)$ & $5(15.15)$ & $6(6.06)$ & 0.564 \\
\hline $50-60$ & $12(10.91)$ & $4(12.13)$ & $19(19.19)$ & \\
\hline $60-70$ & $37(33.64)$ & $11(33.33)$ & 37 (37.37) & \\
\hline $70-80$ & $46(41.82)$ & $13(39.39)$ & $35(35.35)$ & \\
\hline$\geq 80$ & $11(10.00)$ & 0 & $2(2.02)$ & \\
\hline \multicolumn{5}{|l|}{ Sex } \\
\hline Female & $49(44.55)$ & $20(60.61)$ & $44(44.44)$ & 0.202 \\
\hline Male & $61(55.45)$ & $13(39.39)$ & $55(55.56)$ & \\
\hline NSAID use & $51(46.36)$ & $17(51.52)$ & $24(24.24)$ & $<0.001$ \\
\hline \multicolumn{5}{|l|}{ Sydney System Factor Score } \\
\hline Atrophy antrum & $1.221 \pm 0.778$ & $1.160 \pm 0.898$ & $0.986 \pm 0.778$ & 0.254 \\
\hline corpus & $0.755 \pm 0.860$ & $0.429 \pm 0.898$ & $0.613 \pm 0.751$ & 0.140 \\
\hline Metaplasia antrum & $0.633 \pm 0.889$ & $0.515 \pm 0.667$ & $0.443 \pm 0.667$ & 0.411 \\
\hline corpus & $0.284 \pm 0.708$ & $0.156 \pm 0.448$ & $0.122 \pm 0.503$ & 0.116 \\
\hline Mononuclear cell antrum & $1.211 \pm 0.639$ & $1.303 \pm 0.684$ & $1.243 \pm 0.648$ & 0.713 \\
\hline corpus & $1.211 \pm 0.759$ & $1.273 \pm 0.674$ & $1.102 \pm 0.681$ & 0.428 \\
\hline Neutrophil antrum & $0.352 \pm 0.646$ & $0.455 \pm 0.833$ & $0.427 \pm 0.778$ & 0.925 \\
\hline corpus & $0.444 \pm 0.777$ & $0.424 \pm 0.708$ & $0.433 \pm 0.776$ & 0.947 \\
\hline H. pylori antrum & $0.389 \pm 0.783$ & $0.273 \pm 0.626$ & $0.333 \pm 0.660$ & 0.769 \\
\hline corpus & $0.333 \pm 0.641$ & $0.424 \pm 0.969$ & $0.443 \pm 0.841$ & 0.826 \\
\hline
\end{tabular}

Abbreviations: PPI, proton pump inhibitor; H2RA, histamine 2 receptor antagonist; NSAID, non-steroidal anti-inflammatory drug. Bold, statistical significance.

\subsection{Changes in the Updated Sydney System Scores and Association with Drug Use}

Of the 242 patients, the metaplastic histological scores improved in 36 patients and were exacerbated in 27 patients.

Associations between changes in the updated Sydney system scores and drug use are shown in Table 2. Improvements in the atrophy scores of both the antrum and corpus were significantly superior in patients with non-acid suppressant use compared with those of PPI and H2RA users $(p=0.042$, 0.020). Metaplasia scores did not improve in either drug users or non-users, and no significant differences were observed among the groups. Neutrophil, mononuclear cell, and H. pylori scores improved in all groups, but no significant differences in these scores were observed among the groups.

Table 2. Changes in the updated Sydney system score over time by drug use $(n=242)$.

\begin{tabular}{ccccc}
\hline \multirow{2}{*}{ Sydney System Factor } & PPI Use $(\boldsymbol{n}=\mathbf{1 1 0})$ & H2RA Use $(\boldsymbol{n}=\mathbf{3 3 )}$ & $\begin{array}{c}\text { Non-Acid Suppressant } \\
\text { Use }(\boldsymbol{n}=\mathbf{9 9})\end{array}$ & \multirow{2}{*}{$\boldsymbol{p}$ Value } \\
\cline { 2 - 5 } & \multicolumn{2}{c}{ Score Coefficient (Point), Per Year (95\% CI) } & \\
\hline Atrophy antrum & $-0.004(-0.028$ to 0.020$)$ & $-0.011(-0.060$ to 0.039$)$ & $-0.036(-0.059$ to -0.012$)$ & $\mathbf{0 . 0 4 2}$ \\
corpus & $-0.023(-0.045$ to -0.0002$)$ & $-0.021(-0.050$ to 0.009$)$ & $-0.030(-0.052$ to -0.007$)$ & $\mathbf{0 . 0 2 0}$ \\
Metaplasia antrum & $0.014(-0.009$ to 0.036$)$ & $-0.031(-0.069$ to 0.007$)$ & $-0.007(-0.030$ to 0.015$)$ & 0.271 \\
corpus & $-0.003(-0.020$ to 0.013$)$ & $-0.007(-0.033$ to 0.019$)$ & $0.001(-0.012$ to 0.015$)$ & 0.077 \\
Mononuclear cell antrum & $-0.050(-0.064$ to -0.035$)$ & $-0.050(-0.080$ to -0.020$)$ & $-0.055(-0.071$ to -0.039$)$ & 0.815 \\
corpus & $-0.063(-0.080$ to -0.045$)$ & $-0.035(-0.062$ to -0.008$)$ & $-0.051(-0.068$ to -0.034$)$ & 0.204 \\
Neutrophil antrum & $-0.034(-0.049$ to -0.019$)$ & $-0.040(-0.072$ to -0.009$)$ & $-0.053(-0.069$ to -0.037$)$ & 0.961 \\
corpus & $-0.047(-0.065$ to -0.029$)$ & $-0.037(-0.065$ to -0.009$)$ & $-0.050(-0.066$ to -0.034$)$ & 0.350 \\
H. pylori antrum & $-0.038(-0.055$ to -0.020$)$ & $-0.027(-0.052$ to -0.001$)$ & $-0.043(-0.057$ to -0.028$)$ & 0.395 \\
corpus & $-0.040(-0.056$ to -0.023$)$ & $-0.042(-0.082$ to -0.003$)$ & $-0.060(-0.078$ to -0.041$)$ & 0.906 \\
\hline
\end{tabular}

Abbreviations: PPI, proton pump inhibitor; H2RA, histamine 2 receptor antagonist. Bold, statistical significance. 
Associations between changes in the updated Sydney system scores and duration of PPI and H2RA use are shown in Tables 3 and 4. Long-term PPI users showed significantly reduced improvement of corpus atrophy score compared to short-term users $(p=0.016)$. No significant differences in all Sydney system factor scores were observed between long- and short-term H2RA drug use.

Table 3. Subgroup analysis of changes in the updated Sydney system score over time in relation to duration of PPI use.

\begin{tabular}{cccc}
\hline \multirow{2}{*}{ Sydney System Factor } & PPI Long-term Use $(\boldsymbol{n = 8 7 )}$ & PPI Short-term Use $(\boldsymbol{n}=\mathbf{2 3})$ & \multirow{2}{*}{$\boldsymbol{p}$ Value } \\
\cline { 2 - 3 } & \multicolumn{2}{c}{ Score Coefficient (Point), Per Year (95\% CI) } & \\
\hline Atrophy antrum & $-0.002(-0.029$ to 0.026$)$ & $-0.016(-0.075$ to 0.043$)$ & 0.189 \\
corpus & $-0.021(-0.045$ to 0.003$)$ & $-0.027(-0.095$ to 0.041$)$ & $\mathbf{0 . 0 1 6}$ \\
Metaplasia antrum & $0.014(-0.011$ to 0.039$)$ & $0.006(-0.048$ to 0.060$)$ & 0.639 \\
corpus & $-0.0001(-0.017$ to 0.017$)$ & $-0.040(-0.092$ to 0.012$)$ & 0.177 \\
Mononuclear cell antrum & $-0.052(-0.067$ to -0.037$)$ & $-0.036(-0.082$ to 0.009$)$ & 0.957 \\
corpus & $-0.064(-0.084$ to -0.045$)$ & $-0.051(-0.088$ to -0.013$)$ & 0.656 \\
Neutrophil antrum & $-0.028(-0.043$ to -0.012$)$ & $-0.060(-0.102$ to -0.018$)$ & 0.682 \\
corpus & $-0.042(-0.063$ to -0.022$)$ & $-0.070(-0.110$ to -0.030$)$ & 0.777 \\
H. pylori antrum & $-0.032(-0.049$ to -0.014$)$ & $-0.067(-0.128$ to -0.006$)$ & 0.107 \\
corpus & $-0.038(-0.057$ to -0.019$)$ & $-0.044(-0.078$ to -0.010$)$ & 0.631 \\
\hline \multicolumn{2}{c}{ Abbreviations: PPIs, proton pump inhibitors. Bold, statistical significance. } &
\end{tabular}

Table 4. Subgroup analysis of changes in the updated Sydney system score over time in relation to duration of H2RA use.

\begin{tabular}{cccc}
\hline \multirow{2}{*}{ Sydney System Factor } & H2RA Long-term Use $(\boldsymbol{n = 1 6 )}$ & H2RA Short-term Use $(\boldsymbol{n}=\mathbf{1 7})$ & \multirow{2}{*}{$\boldsymbol{p}$ Value } \\
\cline { 2 - 3 } & \multicolumn{2}{c}{ Score Coefficient (Point), Per Year (95\% CI) } & \\
\hline Atrophy antrum & $-0.037(-0.117$ to 0.044$)$ & $0.045(-0.056$ to 0.147$)$ & 0.382 \\
corpus & $-0.034(-0.067$ to -0.001$)$ & $0.005(-0.056$ to 0.066$)$ & 0.108 \\
Metaplasia antrum & $-0.053(-0.100$ to -0.0057$)$ & $-0.022(-0.075$ to 0.031$)$ & 0.296 \\
corpus & $-0.021(-0.066$ to 0.024$)$ & $-0.008(-0.035$ to 0.019$)$ & 0.350 \\
Mononuclear cell antrum & $-0.039(-0.085$ to 0.007$)$ & $-0.078(-0.135$ to -0.022$)$ & 0.567 \\
corpus & $-0.026(-0.070$ to 0.017$)$ & $-0.051(-0.102$ to -0.001$)$ & 0.988 \\
Neutrophil antrum & $-0.034(-0.076$ to 0.009$)$ & $-0.066(-0.129$ to -0.002$)$ & 0.681 \\
corpus & $-0.026(-0.061$ to 0.009$)$ & $-0.063(-0.124$ to -0.003$)$ & 0.692 \\
H. pylori antrum & $-0.023(-0.057$ to 0.010$)$ & $-0.065(-0.144$ to 0.015$)$ & 0.443 \\
corpus & $-0.035(-0.084$ to 0.014$)$ & $-0.038(-0.091$ to 0.015$)$ & 0.633 \\
\hline
\end{tabular}

Abbreviations: H2RA, histamine 2 receptor antagonist.

\subsection{Association between Caudal Related Homeobox Gene (CDX)1 and CDX2 Expression and Changes in Metaplasia and Atrophy Scores}

We selected 63 patients whose metaplasia scores markedly changed after eradication (36 patients with improvement and 27 patients with exacerbation) and investigated changes in Caudal related homeobox gene (CDX)1 and CDX2 expression levels between the initial and final biopsies based on immunohistochemistry, and incomplete metaplasia rate in the same specimens upon hematoxylin and eosin staining.

CDX1 was expressed in 16 of 63 patients (25.4\%). CDX1 expression level significantly decreased in patients showing an improvement in the metaplasia score of the antrum $(0.217$ to $0.033, p<0.001)$ and corpus ( 0.092 to $0.031, p=0.019)$ (Table 5).

CDX2 was expressed in 25 of 63 patients (39.7\%). CDX2 expression level significantly decreased in patients with an improvement in the metaplasia score of the antrum $(0.195$ to $0.01, p<0.001)$ (Table 5).

We also evaluated the changes in incomplete metaplasia prevalence in these specimens. The changes were mostly similar to intestinal metaplasia score improvement/exacerbation, but no statistically significant differences were observed (Table S3). 
In addition, we evaluated CDX1 and CDX2 expressional changes in cases with significant atrophy score changes, but no association with these molecular expression rates was observed (Tables S1 and S2).

Table 5. Association between changes in the metaplasia score and CDX1 and CDX2 expression $(\mathrm{N}=63)$.

\begin{tabular}{|c|c|c|c|}
\hline \multirow{2}{*}{ Metaplasia Change } & \multicolumn{2}{|c|}{ CDX1 Expression Rates, Mean \pm SD } & \multirow{2}{*}{$p$ Value } \\
\hline & Initial assessment & Final assessment & \\
\hline \multicolumn{4}{|l|}{ Improvement } \\
\hline Antrum $(\mathrm{N}=25)$ & $0.217 \pm 0.231$ & $0.033 \pm 0.091$ & $<0.001$ \\
\hline Corpus $(\mathrm{N}=11)$ & $0.092 \pm 0.103$ & $0.008 \pm 0.022$ & 0.019 \\
\hline \multicolumn{4}{|l|}{ Exacerbation } \\
\hline Antrum $(\mathrm{N}=21)$ & $0.059 \pm 0.132$ & $0.100 \pm 0.169$ & 0.128 \\
\hline Corpus $(\mathrm{N}=6)$ & $0.002 \pm 0.005$ & $0.093 \pm 0.105$ & 0.080 \\
\hline \multirow{2}{*}{ Metaplasia Change } & \multicolumn{2}{|c|}{ CDX2 Expression Rate, Mean \pm SD } & $p$ Value \\
\hline & Initial Assessment & Final Assessment & \\
\hline \multicolumn{4}{|l|}{ Improvement } \\
\hline Antrum $(\mathrm{N}=25)$ & $0.195 \pm 0.194$ & $0.031 \pm 0.059$ & $<0.001$ \\
\hline Corpus $(\mathrm{N}=11)$ & $0.128 \pm 0.168$ & $0.069 \pm 0.211$ & 0.401 \\
\hline \multicolumn{4}{|l|}{ Exacerbation } \\
\hline Antrum $(\mathrm{N}=21)$ & $0.060 \pm 0.100$ & $0.114 \pm 0.156$ & 0.079 \\
\hline Corpus $(\mathrm{N}=6)$ & $0.003 \pm 0.007$ & $0.103 \pm 0.155$ & 0.159 \\
\hline
\end{tabular}

Improvement and exacerbation were defined as a decrease and increase, respectively, in the updated Sydney system score between the first and final biopsies. Abbreviations: SD, standard deviation; CDX, Caudal related homeobox gene. Bold, statistical significance.

\section{Discussion}

We showed that $H$. pylori eradication improves histological atrophy and inflammation but does not accelerate the recovery of intestinal metaplasia in our 5.4-year observation period. In particular, PPI or H2RA use may inhibit the improvement of gastric atrophy compared with non-acid suppressant users. Therefore, gastric atrophy may be regulated by molecularly distinct mechanisms to that of intestinal metaplasia (i.e., aberrant expression of CDX proteins). In other words, improvement of gastric atrophy after H. pylori eradication may be independent of changes in intestinal metaplasia [15-20], which seems to be a more sustained, stable reprogramming event of the gastric glands. In our study, the expression of CDX1 was more closely associated with changes in the intestinal metaplasia score than that of CDX2. In mice, ectopic expression of CDX1 and CDX2 in the stomach induces intestinal metaplasia, but expression of either CDX genes alone does not induce expression of the other gene [21]. Therefore, CDX1 and CDX2 may be independently regulated. Our findings suggest that changes in CDX1 expression might precede changes in CDX2 expression.

The finding that eradication resulted in improvement of atrophy in the corpus is consistent with a previous meta-analysis of short-term observational studies [22] and a long-term observational study in Japan [23] but inconsistent with a randomized controlled trial in Korea [15] and a long-term observational study [24]. By contrast, the lack of improvement in intestinal metaplasia after eradication is inconsistent with some previous studies $[15,22]$. This may be explained by the different scoring systems used for evaluation of atrophy and metaplasia. In addition, baseline histological severities of atrophy and intestinal metaplasia were different. There are also differences in regions and countries, races and, presumably, $H$. pylori strains among these studies, all of which may affect outcomes.

Previously, Annibale et al. reported that $H$. pylori eradication improved gastric atrophy only in a subset of patients whose serum gastrin level became normal during the observation period, while the remaining patients showed sustained gastric atrophy with elevated serum gastrin [25]. A reasonable interpretation of these results would be that restoration of normal acid secretion in improved patients suppresses the elevation of serum gastrin in a negative feedback manner. Nonetheless, it is possible 
that an elevated level of serum gastrin, perhaps due to the intake of acid-suppressing drugs, delays the recovery of gastric atrophy. In mice, hypergastrinemia induces gastric atrophy, metaplasia and, eventually, cancer [26], and these changes are accelerated by the administration of PPIs [27]. Long-term PPI use also promotes gastric atrophy and cancer development in Helicobacter-infected Mongolian gerbils [28]. In humans, long-term use of PPIs has been reported to worsen corpus atrophic gastritis in the patients infected with $H$. pylori $[29,30]$. In any case, prior epidemiological retrospective cohort studies [11,12] and our current findings suggest that PPI use may prolong gastric atrophy after eradication, which may increase the risk for gastric cancer in post-eradicated patients [1].

Although $H$. pylori infection appears to be inversely associated with gastroesophageal reflux disease due to low acid secretion from the atrophic stomach, certain populations of $H$. pylori-infected patients continue to suffer from acid reflux symptoms. In our cohort, the gastrointestinal symptom rating scale (GSRS) scores of upper abdominal pain, heartburn, abdominal distention, and bloating were temporarily decreased (improved) after H. pylori eradication (Tables S4 and S5), while these scores eventually returned to their original levels after the mean 5-year observation period. Thus, PPIs or H2RAs are often prescribed for patients with chronic acid reflux symptoms after eradication; however, our data suggest that the use of H2RAs or PPIs may be more cautiously considered given the potential effects on gastric atrophy and subsequent cancer risk.

The strengths of this study are the long-term, comprehensive assessment of clinical, endoscopic, histological, and molecular findings. Our analysis is quantitative and has extensively investigated the long-term effects of PPI and H2RA use following H. pylori eradication. However, this study also had limitations. Firstly, although we conducted long-term follow-up, the analyses were not prospective. The number of biopsy specimens and the observation period were limited, and some patients were lost to follow-up; secondly, our immunohistochemical stain data were limited to certain patients, associated with potential selection bias; thirdly, our sample size might be too small to reliably evaluate further associations between duration of drug use and histological score changes; fourthly, NSAIDs were more commonly taken in PPI and H2RA users than in non-drug users, and it might act as a confounder and affect the outcome; finally, our cohort lacked consideration of confounding factors such as smoking status, alcohol intake, and diet.

In conclusion, gastric atrophy but not intestinal metaplasia may be improved in patients who underwent successful H. pylori eradication, especially in patients with non-acid-suppressing drug use. Long-term acid suppression therapy might be associated with sustained gastric atrophy following eradication.

\section{Methods}

\subsection{Study Design and Setting}

We collected consecutive data on patients who underwent $H$. pylori eradication therapy and gastric cancer surveillance at Tokyo University Hospital from 1996 to 2015 (Figure 1A, study flow chart). We performed retrospective cohort analyses of these data. This study was approved by the Institutional Review Board of the University of Tokyo (no. 2058-2, 17 November 2017) and was performed in accordance with an assurance filed with and approved by the Japanese Ministry of Health, Labour, and Welfare.

\subsection{Participants}

Patients who were positive for $H$. pylori infection and atrophic gastritis by endoscopy, and had undergone successful eradication therapy, were eligible for inclusion in this study. H. pylori infection was diagnosed by urea breath test, culture, rapid urease test, or serum antibody test. An exclusion criterion was a non-eradication assessment by urea breath test. 


\subsection{Eradication Treatment and Assessment}

As the first-line treatment, amoxicillin $(750 \mathrm{mg})$, clarithromycin $(500 \mathrm{mg})$, and the PPI lansoprazole (30 mg) were administered twice daily for 7 days. As the second-line treatment, metronidazole $(250 \mathrm{mg})$, amoxicillin (750 mg), and the PPI lansoprazole (30 mg) were given twice daily for 7 days. The H. pylori eradication status was determined based on the urea breath test.

After eradication, all patients underwent upper gastrointestinal endoscopy evaluation every 1 year up to 2017. Data were censored at the time of the last endoscopic examination or the last hospital visit for patients who were lost to follow-up or who withdrew from the study (Figure 1B, study time course).

\subsection{Upper Gastrointestinal Endoscopy and Histological Examination}

All upper gastrointestinal endoscopies were performed using an electronic video endoscope (Olympus Medical System, Tokyo, Japan).

An endoscopic biopsy was performed to evaluate intestinal metaplasia, atrophy, neutrophil and mononuclear cell infiltration, and H. pylori scores according to the updated Sydney system [31]. These parameters were scored as follows: normal, 0 points; mild, 1 point; moderate, 2 points; and marked, 3 points. Biopsy specimens were obtained from the antrum and the middle corpus of the greater curvature based on our previous works [1,12,32]. Incomplete metaplasia was also evaluated by hematoxylin and eosin staining. Histological findings were assessed and confirmed by two experienced pathologists without any disagreement.

\subsection{Outcomes and Variables}

The primary outcome was change in the intestinal metaplasia, atrophy, neutrophil and mononuclear cell infiltration, and $\mathrm{H}$. pylori scores in patients with PPI, $\mathrm{H}_{2}$ receptor agonist (H2RA), and non-acid suppressant use. The secondary outcome was change in these scores in patients according to the duration of acid suppressing therapy. We also evaluated associations in changes of histology and CDX1 and CDX2 expression between the first and final biopsies in the cases whose intestinal metaplasia or atrophy score changed during study periods. For intestinal metaplasia and atrophy, improvement and exacerbation were defined as a decrease and increase, respectively, in the updated Sydney system score between the first and final biopsies.

We evaluated age, sex, medication use (PPIs, H2RAs, and non-steroidal anti-inflammatory drugs (NSAIDs)), and acid-reflux symptoms. Age was categorized into five groups. Use of PPIs, H2RAs, and NSAIDs was defined as regular oral administration for at least 30 days during the follow-up period. Based on an appropriate receiver operating characteristic curve model for predicting atrophy execration, duration of PPIs and H2RAs was categorized as follows: PPI short-term use, <90 days; PPI long-term use, $\geq 90$ days; H2RA short-term use, $<485$ days; H2RA long-term use, $\geq 485$ days. Use of NSAIDs included low-dose aspirin use. Acid-reflux symptoms were evaluated using the upper gastrointestinal related gastrointestinal symptom rating scale (GSRS): upper abdominal pain, heartburn, acid reflux, abdominal distention, hunger pain, rumbling, bloating, and burning, administered as a seven-point Likert scale self-reported questionnaire [33].

\subsection{Immunohistochemical Stain Analyses of CDX1 and CDX2 Expression}

Sections (3 mm thick) were deparaffinized, rehydrated in phosphate-buffered saline (PBS), placed in $10 \mathrm{mM}$ citrate buffer ( $\mathrm{pH}$ 6.0), and heated to $120^{\circ} \mathrm{C}$ for $5 \mathrm{~min}$ to recover antigenicity. Sections were preincubated with blocking buffer ( $3 \%$ hydrogen peroxide) for $5 \mathrm{~min}$ at room temperature. The primary anti-CDX1 polyclonal antibody (1:100; BioGenex, Fremont, CA, USA) and anti-CDX2 monoclonal antibody (1:100; Abcam, Cambridge, UK) were diluted in PBS and incubated with the sections overnight at $4{ }^{\circ} \mathrm{C}$. Sections were then washed in PBS and incubated with Histofine Simple Stain Max-Po (Multi) (Nichirei Biosciences Inc., Tokyo, Japan). After development with 3,3,9-diaminobenzidine tetrahydrochloride (DAB Substrate Kit, Nichirei Biosciences), sections were counterstained with 
hematoxylin and viewed under a light microscope. CDX1 and CDX2 expression were assessed as proportions of CDX1- and CDX2-expressing gastric glands by one researcher (RN) (Figure 2).

\subsection{Statistical Analyses}

We calculated the coefficients and 95\% confidence intervals (CIs) of annual changes in the atrophy, intestinal metaplasia, mononuclear cell and neutrophil infiltration, and H. pylori scores in biopsies of the antrum and middle corpus of the greater curvature and compared these scores among PPI, H2Rs, and non-acid suppressant users based on a generalized linear mixed model. We also compared the coefficients and $95 \%$ CIs of the annual changes of atrophy, intestinal metaplasia, mononuclear cell and neutrophil infiltration, and H. pylori scores between short- and long-term users of PPIs and H2RAs using a generalized linear mixed model. In addition, we evaluated changes between the initial and final CDX1 and CDX2 expression levels using paired $t$-tests in each metaplasia and atrophy change subgroup. Furthermore, we evaluated changes between the initial and final incomplete metaplasia rates using chi-squared tests in each metaplasia subgroup. We evaluated changes in the GSRS before and 3 months after eradication and changes in the GSRS during all follow-up periods using paired $t$-tests. Statistical analyses were performed using SAS software v. 9.4 (SAS Institute, Cary, NC, USA). A $p$-value $<0.05$ was considered to indicate statistical significance.

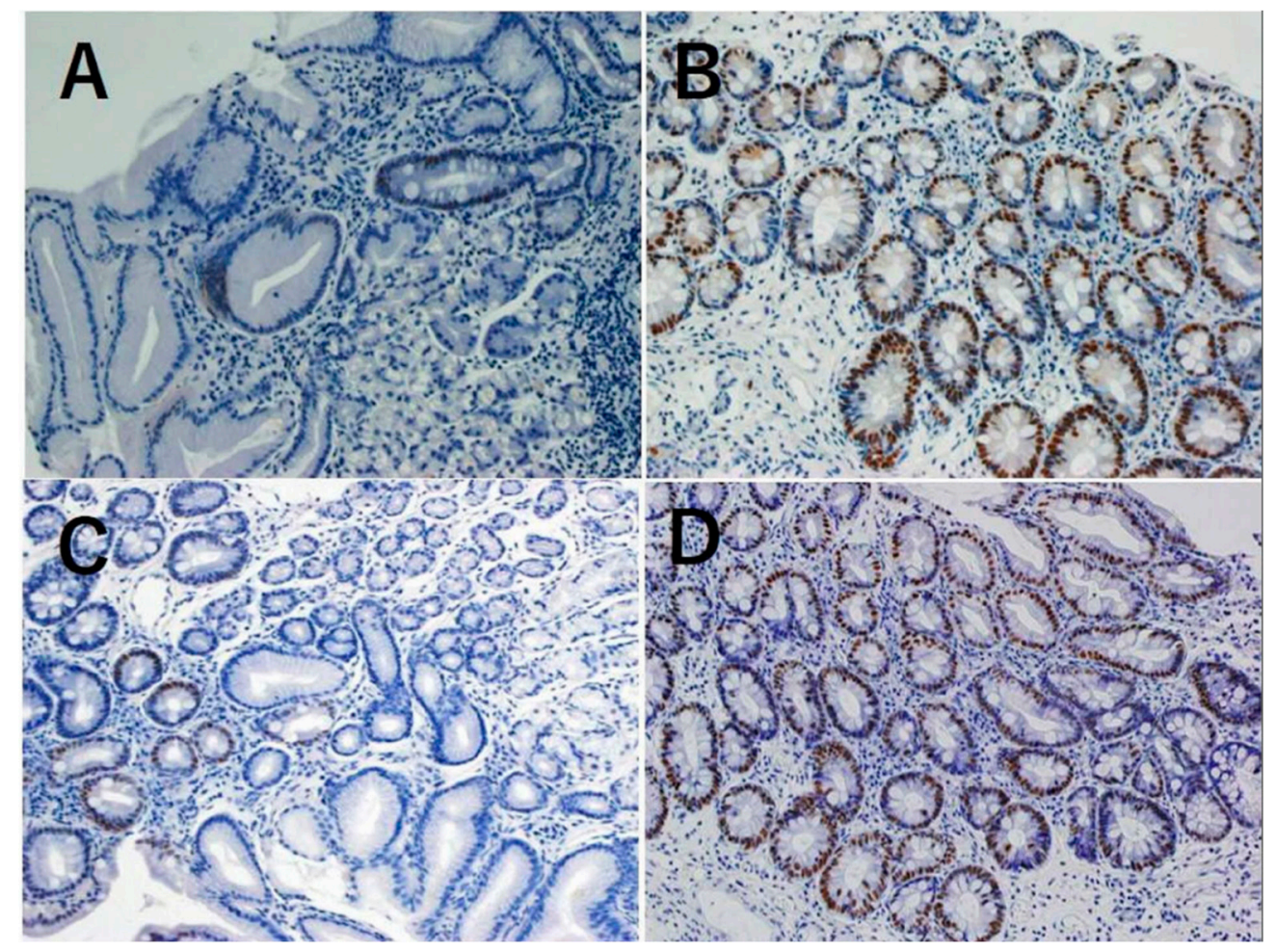

Figure 2. Immunohistochemical stain of Caudal related homeobox gene (CDX)1 and CDX2 expression in the biopsied gastric mucosa: low positive $(<50 \%)$ for CDX1 100× (A); high positive ( $>50 \%)$ for CDX1

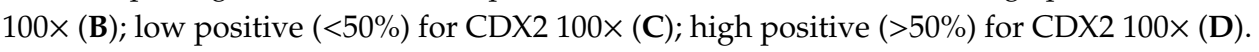

Supplementary Materials: Supplementary materials can be found at http://www.mdpi.com/1422-0067/20/8/1913/s1.

Author Contributions: R.N. and Y.H. (Yoku Hayakawa) are equally corresponding authors in terms of their contribution to the study design and manuscript. R.N., Y.H. (Yoku Hayakawa), Y.H. (Yoshihiro Hirata), K.O., and A.Y. performed endoscopic biopsies. R.N. and Y.H. (Yoku Hayakawa) conducted all immunohistochemical stain analyses. T.U. conducted histological evaluation of biopsy samples. R.N. performed all statistical analyses. K.O., M.F. (Mitsuhiro Fujishiro), A.Y., T.U., M.K., M.F. (Masashi Fukayama), and K.K. advised on the study and contributed to manuscript preparation. All authors have read and approved the final manuscript. 
Funding: This study was supported by KAKENHI Grants-in-Aid for Scientific Research (grant numbers 17K15928 (R.N.), 16H06749, 17H05081 (Y.H. (Yoku Hayakawa)), and 17K03946 Y.H. (Yoshihiro Hirata)), the Asahi Life Foundation (R.N.), P-CREATE from AMED, and the Advanced Research and Development Programs for Medical Innovation (PRIME) (Y. Hayakawa). The funding agents had no role in the design of the study, data collection and analyses, decision to publish, or preparation of the manuscript.

Acknowledgments: We thank the patients and their families. We also thank Tomohiro Shinozaki for help with statistical analyses and Yukiko Otori for assistance with data management.

Conflicts of Interest: The authors declare no conflict of interest.

\title{
Abbreviations
}

\author{
H. pylori Helicobacter pylori \\ PPIs proton pump inhibitors \\ H2RAs $\quad \mathrm{H}_{2}$ receptor antagonists
}

\section{References}

1. Shichijo, S.; Hirata, Y.; Niikura, R.; Hayakawa, Y.; Yamada, A.; Ushiku, T.; Fukayama, M.; Koike, K. Histologic intestinal metaplasia and endoscopic atrophy are predictors of gastric cancer development after helicobacter pylori eradication. Gastrointest. Endosc. 2016, 84, 618-624. [CrossRef]

2. Murakami, K.; Kodama, M.; Nakagawa, Y.; Mizukami, K.; Okimoto, T.; Fujioka, T. Long-term monitoring of gastric atrophy and intestinal metaplasia after helicobacter pylori eradication. Clin. J. Gastroenterol. 2012, 5, 247-250. [CrossRef]

3. Kodama, M.; Murakami, K.; Okimoto, T.; Abe, T.; Nakagawa, Y.; Mizukami, K.; Uchida, M.; Inoue, K.; Fujioka, T. Helicobacter pylori eradication improves gastric atrophy and intestinal metaplasia in long-term observation. Digestion 2012, 85, 126-130. [CrossRef]

4. Toyokawa, T.; Suwaki, K.; Miyake, Y.; Nakatsu, M.; Ando, M. Eradication of helicobacter pylori infection improved gastric mucosal atrophy and prevented progression of intestinal metaplasia, especially in the elderly population: A long-term prospective cohort study. J. Gastroenterol. Hepatol. 2010, 25, 544-547. [CrossRef]

5. Rocco, A.; Suriani, R.; Cardesi, E.; Venturini, I.; Mazzucco, D.; Nardone, G. Gastric atrophy and intestinal metaplasia changes 8 years after helicobacter pylori eradication. A blind, randomised study. Minerva Gastroenterol. Dietol. 2002, 48, 175-178. [PubMed]

6. Fukase, K.; Kato, M.; Kikuchi, S.; Inoue, K.; Uemura, N.; Okamoto, S.; Terao, S.; Amagai, K.; Hayashi, S.; Asaka, M.; et al. Effect of eradication of helicobacter pylori on incidence of metachronous gastric carcinoma after endoscopic resection of early gastric cancer: An open-label, randomised controlled trial. Lancet 2008, 372, 392-397. [CrossRef]

7. Kong, Y.J.; Yi, H.G.; Dai, J.C.; Wei, M.X. Histological changes of gastric mucosa after helicobacter pylori eradication: A systematic review and meta-analysis. World J. Gastroenterol. 2014, 20, 5903-5911. [CrossRef]

8. Testoni, P.A.; Masci, E.; Marchi, R.; Guslandi, M.; Ronchi, G.; Tittobello, A. Gastric cancer in chronic atrophic gastritis. associated gastric ulcer adds no further risk. J. Clin. Gastroenterol. 1987, 9, 298-302. [CrossRef]

9. Uemura, N.; Okamoto, S.; Yamamoto, S.; Matsumura, N.; Yamaguchi, S.; Yamakido, M.; Taniyama, K.; Sasaki, N.; Schlemper, R.J. Helicobacter pylori infection and the development of gastric cancer. N. Engl. J. Med. 2001, 345, 784-789. [CrossRef] [PubMed]

10. Waldum, H.L.; Fossmark, R. Types of gastric carcinomas. Int. J. Mol. Sci. 2018, 19, 4109. [CrossRef] [PubMed]

11. Waldum, H.L.; Hauso, O.; Sordal, O.F.; Fossmark, R. Gastrin may mediate the carcinogenic effect of helicobacter pylori infection of the stomach. Dig. Dis. Sci. 2015, 60, 1522-1527. [CrossRef]

12. Cheung, K.S.; Chan, E.W.; Wong, A.Y.S.; Chen, L.; Wong, I.C.K.; Leung, W.K. Long-term proton pump inhibitors and risk of gastric cancer development after treatment for helicobacter pylori: A population-based study. Gut 2018, 67, 28-35. [CrossRef]

13. Niikura, R.; Hayakawa, Y.; Hirata, Y.; Yamada, A.; Fujishiro, M.; Koike, K. Long-term proton pump inhibitor use is a risk factor of gastric cancer after treatment for helicobacter pylori: A retrospective cohort analysis. Gut 2018, 67, 1908-1910. [CrossRef] [PubMed] 
14. Kinoshita, H.; Hayakawa, Y.; Koike, K. Metaplasia in the stomach-precursor of gastric cancer? Int. J. Mol. Sci. 2017, 18, 2063. [CrossRef] [PubMed]

15. Spence, A.D.; Cardwell, C.R.; McMenamin, U.C.; Hicks, B.M.; Johnston, B.T.; Murray, L.J.; Coleman, H.G. Adenocarcinoma risk in gastric atrophy and intestinal metaplasia: A systematic review. BMC Gastroenterol. 2017, 17, 157. [CrossRef]

16. Dixon, M.F.; Genta, R.M.; Yardley, J.H.; Correa, P. Classification and grading of gastritis. The updated sydney system. international workshop on the histopathology of gastritis, houston 1994. Am. J. Surg. Pathol. 1996, 20, 1161-1181. [CrossRef] [PubMed]

17. Shichijo, S.; Hirata, Y.; Sakitani, K.; Yamamoto, S.; Serizawa, T.; Niikura, R.; Watabe, H.; Yoshida, S.; Yamada, A.; Yamaji, Y. Distribution of intestinal metaplasia as a predictor of gastric cancer development. J. Gastroenterol. Hepatol. 2015, 30, 1260-1264. [CrossRef] [PubMed]

18. Svedlund, J.; Sjodin, I.; Dotevall, G. GSRS-A clinical rating scale for gastrointestinal symptoms in patients with irritable bowel syndrome and peptic ulcer disease. Dig. Dis. Sci. 1988, 33, 129-134. [CrossRef]

19. Choi, I.J.; Kook, M.C.; Kim, Y.I.; Cho, S.J.; Lee, J.Y.; Kim, C.G.; Park, B.; Nam, B.H. Helicobacter pylori therapy for the prevention of metachronous gastric cancer. N. Engl. J. Med. 2018, 378, 1085-1095. [CrossRef]

20. Kwon, Y.J.; Kim, N.; Baek, S.M.; Lee, H.S.; Lee, J.; Hwang, Y.J.; Yoon, H.; Shin, C.M.; Park, Y.S.; Kim, J.W.; et al. The prevalence of histologic atrophy and intestinal metaplasia in the corpus has decreased over 15 years in females in the korean population. Helicobacter 2019. [CrossRef]

21. Hwang, Y.J.; Kim, N.; Lee, H.S.; Lee, J.B.; Choi, Y.J.; Yoon, H.; Shin, C.M.; Park, Y.S.; Lee, D.H. Reversibility of atrophic gastritis and intestinal metaplasia after helicobacter pylori eradication-A prospective study for up to 10 years. Aliment. Pharmacol. Ther. 2018, 47, 380-390. [CrossRef]

22. Rokkas, T.; Pistiolas, D.; Sechopoulos, P.; Robotis, I.; Margantinis, G. The long-term impact of helicobacter pylori eradication on gastric histology: A systematic review and meta-analysis. Helicobacter 2007, 12 (Suppl. 2), 32-38. [CrossRef]

23. Wang, J.; Xu, L.; Shi, R.; Huang, X.; Li, S.W.; Huang, Z.; Zhang, G. Gastric atrophy and intestinal metaplasia before and after helicobacter pylori eradication: A meta-analysis. Digestion 2011, 83, 253-260. [CrossRef] [PubMed]

24. Kong, P.; Wu, R.; Liu, X.; Liu, J.; Chen, S.; Ye, M.; Yang, C.; Song, Z.; He, W.; Yin, C.; et al. The effects of anti-inflammatory drug treatment in gastric cancer prevention: An update of a meta-analysis. J. Cancer 2016, 7, 2247-2257. [CrossRef] [PubMed]

25. Mutoh, H.; Sakurai, S.; Satoh, K.; Osawa, H.; Hakamata, Y.; Takeuchi, T.; Sugano, K. Cdx1 induced intestinal metaplasia in the transgenic mouse stomach: Comparative study with Cdx2 transgenic mice. Gut 2004, 53, 1416-1423. [CrossRef] [PubMed]

26. Kodama, M.; Murakami, K.; Okimoto, T.; Sato, R.; Uchida, M.; Abe, T.; Shiota, S.; Nakagawa, Y.; Mizukami, K.; Fujioka, T. Ten-year prospective follow-up of histological changes at five points on the gastric mucosa as recommended by the updated sydney system after helicobacter pylori eradication. J. Gastroenterol. 2012, 47, 394-403. [CrossRef] [PubMed]

27. Mera, R.M.; Bravo, L.E.; Camargo, M.C.; Bravo, J.C.; Delgado, A.G.; Romero-Gallo, J.; Yepez, M.C.; Realpe, J.L.; Schneider, B.G.; Morgan, D.R.; et al. Dynamics of helicobacter pylori infection as a determinant of progression of gastric precancerous lesions: 16-year follow-up of an eradication trial. Gut 2018, 67, 1239-1246. [CrossRef] [PubMed]

28. Annibale, B.; Di Giulio, E.; Caruana, P.; Lahner, E.; Capurso, G.; Bordi, C.; Delle Fave, G. The long-term effects of cure of helicobacter pylori infection on patients with atrophic body gastritis. Aliment. Pharmacol. Ther. 2002, 16, 1723-1731. [CrossRef] [PubMed]

29. Chen, D.; Zhao, C.M.; Dockray, G.J.; Varro, A.; Van Hoek, A.; Sinclair, N.F.; Wang, T.C.; Koh, T.J. Glycine-extended gastrin synergizes with gastrin 17 to stimulate acid secretion in gastrin-deficient mice. Gastroenterology 2000, 119, 756-765. [CrossRef]

30. Takaishi, S.; Cui, G.; Frederick, D.M.; Carlson, J.E.; Houghton, J.; Varro, A.; Dockray, G.J.; Ge, Z.; Whary, M.T.; Rogers, A.B.; et al. Synergistic inhibitory effects of gastrin and histamine receptor antagonists on helicobacter-induced gastric cancer. Gastroenterology 2005, 128, 1965-1983. [CrossRef]

31. Hagiwara, T.; Mukaisho, K.; Nakayama, T.; Sugihara, H.; Hattori, T. Long-term proton pump inhibitor administration worsens atrophic corpus gastritis and promotes adenocarcinoma development in mongolian gerbils infected with helicobacter pylori. Gut 2011, 60, 624-630. [CrossRef] [PubMed] 
32. Kuipers, E.J.; Uyterlinde, A.M.; Pena, A.S.; Hazenberg, H.J.; Bloemena, E.; Lindeman, J.; Klinkenberg-Knol, E.C.; Meuwissen, S.G. Increase of helicobacter pylori-associated corpus gastritis during acid suppressive therapy: Implications for long-term safety. Am. J. Gastroenterol. 1995, 90, 1401-1406. [PubMed]

33. Kuipers, E.J.; Lundell, L.; Klinkenberg-Knol, E.C.; Havu, N.; Festen, H.P.; Liedman, B.; Lamers, C.B.; Jansen, J.B.; Dalenback, J.; Snel, P.; et al. Atrophic gastritis and helicobacter pylori infection in patients with reflux esophagitis treated with omeprazole or fundoplication. N. Engl. J. Med. 1996, 334, 1018-1022. [CrossRef] [PubMed]

(C) 2019 by the authors. Licensee MDPI, Basel, Switzerland. This article is an open access article distributed under the terms and conditions of the Creative Commons Attribution (CC BY) license (http://creativecommons.org/licenses/by/4.0/). 